\title{
RECENT AND HISTORICAL SOLAR PROTON EVENTS
}

\author{
M. A. SHEA and D. F. SMART \\ Space Physics Division, Geophysics Directorate/Phillips Laboratory, Hanscom Air Force Base \\ Bedford, Massachusetts 01731-5000 USA
}

\begin{abstract}
A study of the solar proton event data between 1954 and 1986 indicates that the large fluence events at the Earth are usually associated with a sequence of solar activity and related geomagnetic storms. This association appears to be useful to infer the occurrence of major fluence proton events extending back to 1934, albeit in a non-homogeneous manner. We discuss the possibility of identifying major solar proton events prior to 1934 , using geomagnetic records as a proxy.
\end{abstract}

\section{INTRODUCTION}

Solar-flare-generated protons impacting the top of the atmosphere account for some of the total radiocarbon inventory. Lingenfelter and Ramaty (1970) have estimated that the solar flare production during the 19 th solar cycle was between $6 \%$ and $14 \%$ of the solar-cycle averaged galactic cosmic-ray production rate. We assume that the source of these protons is associated, in some way, with the occurrence of a solar flare, although a unique acceleration process has not been identified.

Solar-flare accelerated particles travel along the interplanetary magnetic field and impact the Earth's atmosphere principally in the polar regions. Occasionally, the Sun accelerates particles into the $\mathrm{GeV}$ energy range; these particles can penetrate the geomagnetic shielding to lower latitudes and, hence, are more effective to ${ }^{14} \mathrm{C}$ production.

Approximately half of the energy of the solar flare is in the interplanetary shock that travels to the Earth's orbit 1 or 2 days after the flare. Under favorable circumstances, particularly during a sequence of solar activity, the shock re-accelerates the ambient solar particle population, thus modifying the observed particle spectrum from what was released into the interplanetary medium during the original flare process.

When the interplanetary shock impacts the Earth's magnetosphere, the structure of the magnetosphere is altered. Often, depending on the direction of the interplanetary magnetic field vectors, a major geomagnetic storm will occur. The current systems involved in the geomagnetic storm can substantially lower the geomagnetic cutoff rigidity (Flückiger, Smart \& Shea 1986). The occurrence of mid- and low-latitude aurorae is evidence of a major expansion of the Earth's polar caps, which allows solar particles to penetrate much lower in latitude than during quiescent geomagnetic conditions. Figure 1 represents the major solar-flare emissions and the relative time intervals that these emissions take to travel to the orbit of the Earth.

Using data acquired by both spacecraft and ground-based techniques, we have assembled a list of over 200 significant solar proton events that have reached the Earth between 1954 and 1986 . We would like to suggest a method of identifying some of the major proton events over the past century, using a combination of solar flare, solar particle and geomagnetic records.

\section{SOLAR PROTON DATA BASE}

Although the first directly measured solar proton event occurred in 1942, it was not until the International Geophysical Year in 1957-1958 that solar proton events could be routinely identified. Even then, the identification of solar proton events was limited to the major events that could be 


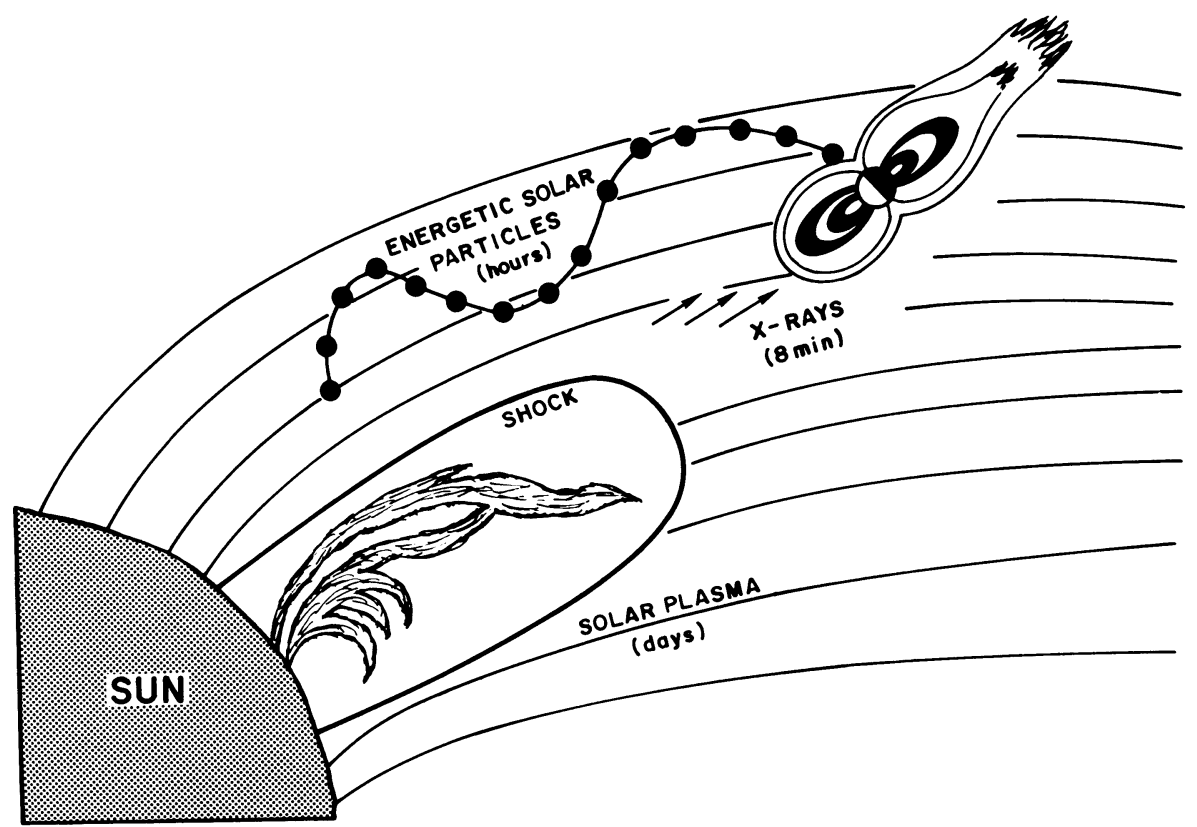

Fig. 1. Pictorial representation of solar emissions from a solar flare. The time intervals that the various emissions take to reach the Earth are also shown.

detected by various ground-based techniques. Routine spacecraft measurements were initiated in 1965; since that time, solar particles have been almost continuously monitored via Earth-orbiting or geosynchronous satellites.

The most homogeneous solar proton data base has been obtained from the cosmic radiation records. From 1934, ionization chambers, and, since 1951, neutron monitors, recorded occasional increases in the cosmic-ray intensity that were associated with the release of $\mathrm{GeV}$ protons from the Sun. From 1956 until mid-1991, neutron monitor measurements have detected 48 relativistic solar particle events at the Earth. These events include protons with energies above $500 \mathrm{MeV}$; in some cases, the energy of the solar protons exceed $10 \mathrm{GeV}$. Figure 2 illustrates the occurrence of these events as a function of time. Although most of the high-energy events occur during periods of enhanced solar activity, as represented by the sunspot number, they can occur even during solar minimum.

Spacecraft observations of solar proton events essentially start near the beginning of the 20th solar cycle. We have examined the relativistic solar proton event data for the 19th solar cycle, and have assembled a data base equivalent to the satellite-sensed data base of the 20th and 21st solar cycles. Thus, our data base is homogeneous for three solar cycles. We identified all events with protons $>10 \mathrm{MeV}$ having a peak flux of $>10$ protons $/\left(\mathrm{cm}^{2}\right.$-sec-ster $)$. Shea and Smart (1990a) list these events, together with the peak proton flux and fluence.

We then separated the events by solar cycle, starting with the month of sunspot minimum, to see if there was an identifiable pattern between the cycles. These results are shown in Figure 3, where the 12-month average of the sunspot number is plotted as histograms for each cycle, and the proton events for each 12-month period is shown. Figure 3 also shows a plot of the number of proton events per year $v s$. the yearly average sunspot number. Other than an increase in the number of events during the maximum in solar activity, there does not appear to be a predictable or repeatable pattern between solar proton event occurrence and the solar activity cycle (as measured by the sunspot number). 

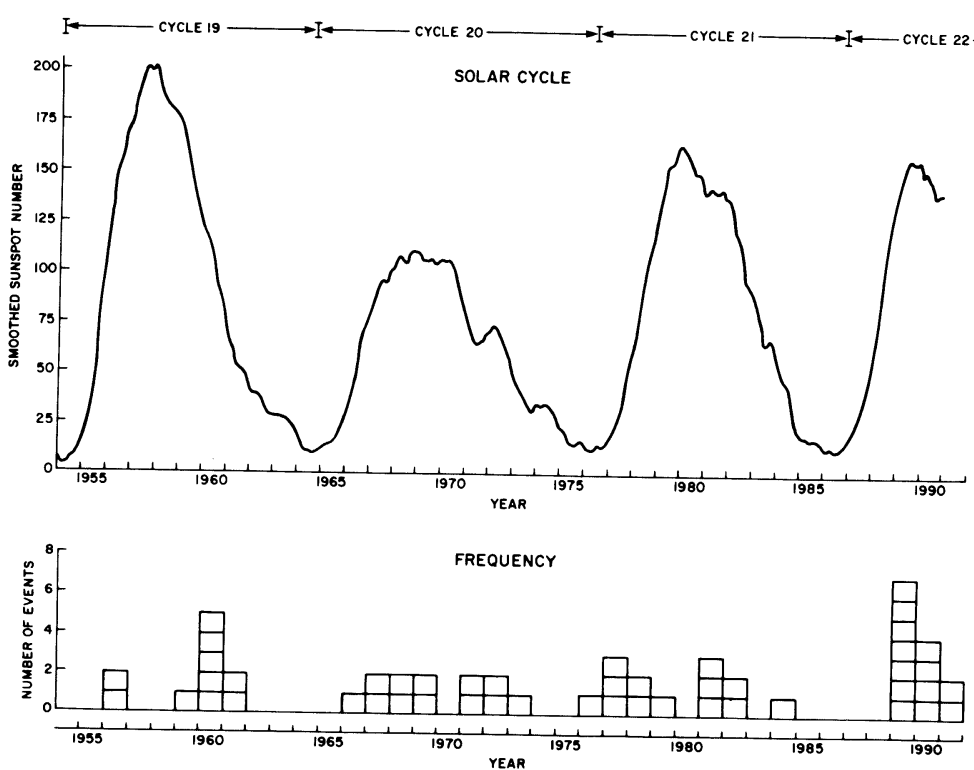

Fig. 2. Relativistic solar proton events observed since 1955. Top - the smoothed sunspot number; Bottom - the number of high energy solar proton events each year.
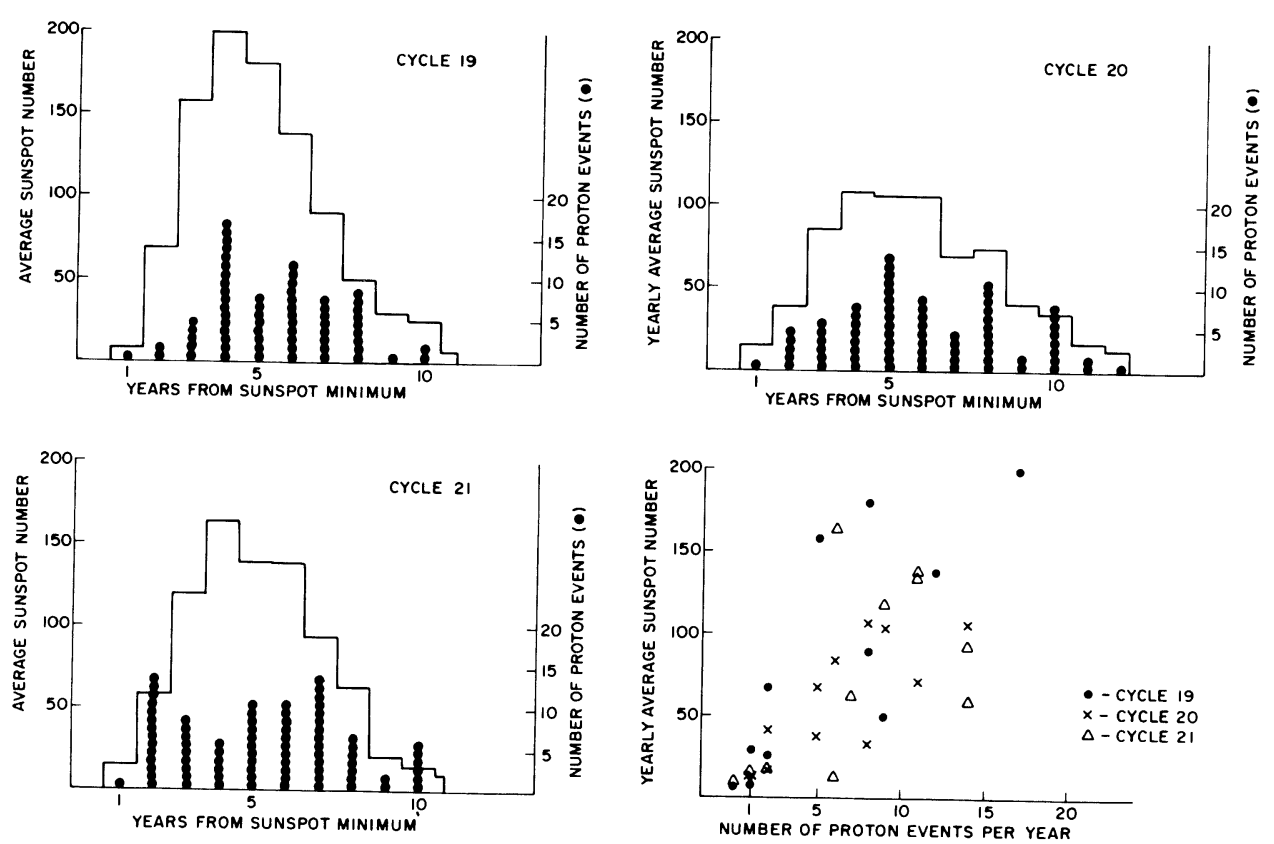

Fig. 3. The number of significant discrete solar proton events for each 12 -month period after solar minimum (•) and the 12-month mean sunspot number for the corresponding period (histograms) for solar cycles 19-21. A plot of the number of proton events per year $v s$. the yearly average sunspot number is shown in the lower right hand section. 


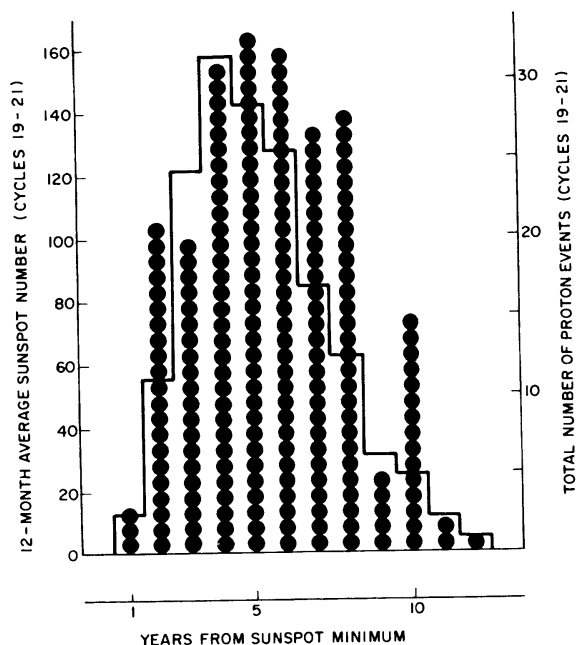

Fig. 4. Summation of significant discrete solar proton events for cycles 19-21 (•) and the corresponding 12-month average sunspot number (histogram). The data are organized in 12-month periods, beginning with the month after sunspot minimum, as defined by the statistically smoothed sunspot number.

In combining these results for the three solar cycles into one figure, we obtain the distribution shown in Figure 4. From this, we conclude that the majority of significant solar proton events occurs from the 2 nd through the 10 th years of the solar cycle.

\section{SOLAR PROTON FLUENCE}

Although most solar particle events are measured in terms of peak proton flux, the fluence is more appropriate for ${ }^{14} \mathrm{C}$ production. Feynman et al. (1990) show that the fluence for events in solar cycles 19-21 all fit in one continuous log-normal distribution. Shea and Smart (1990a) indicate that it is not always possible to identify the fluence on an event-by-event basis, because many events occur in episodes of activity. In these cases, the fluence is determined by summing the flux throughout the entire period. An example of a major episode of activity was August 1972, when a series of flares from one solar region, as it traversed the solar disk, produced one-half the proton fluence above $10 \mathrm{MeV}$ for the entire 11-year solar cycle.

\section{EVENTS IN SOLAR CYCLE 22}

The present solar cycle, which started in October 1986, has been unexpectedly active, with respect to the generation of major solar proton events in both the energy content, flux and fluence. This reinforces the suggestion that we and other researchers have made that the first two solar cycles of the space era may be atypical cycles rather than normal cycles. This suggestion was first published by Goswami et al. (1988), who analyzed the spectral characteristics of solar proton events responsible for the generation of radio nuclei. These authors concluded that the spectral characteristics observed in the 20th and 21st solar cycles were different than observed in the 19th solar cycle. Shea and Smart (1990b) also suggested that the relativistic solar proton events of the 22 nd solar cycle were similar to those in the 17-19th cycles with large fluxes and long durations; the relativistic proton events in the 20 th and 21 st solar cycles were generally smaller events of very short duration. Figure 5 illustrates the difference in duration of two relativistic solar proton events. On 7 May 1978 was the largest event of either the 20th or 21st solar cycle; the high energy solar proton flux, as measured at the Kerguelen Island neutron monitor, lasted for just over two hours. On 29 September 1989 was the largest event of the 22nd solar cycle, to date, and is comparable to the events in November 1960, as well as events measured by ionization chambers prior to 1955 . 


\section{Kerguelen Island}

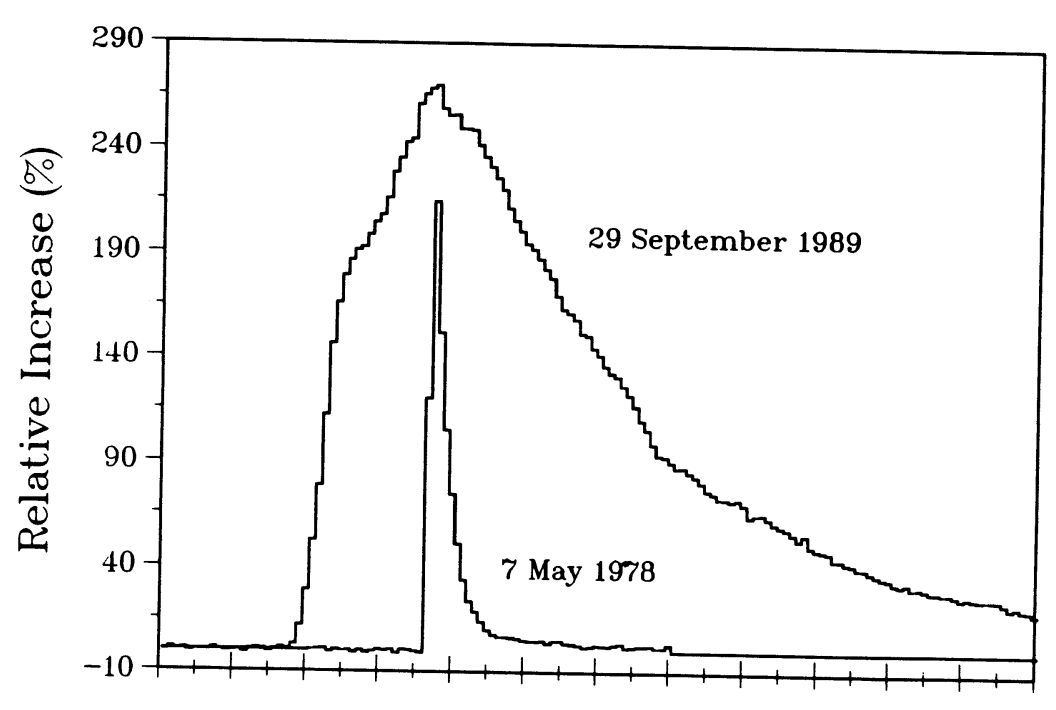

Hour

Fig. 5. The duration of the largest relativistic solar proton event of the 22nd solar cycle (29 September 1989) compared with the largest event of the 20th and 21st solar cycles (7 May 1978). The Kerguelen Island neutron monitor is located at $49.35^{\circ}$ $\mathrm{S}, 70.27^{\circ} \mathrm{E}$ and measures the cosmic radiation above $\sim 500 \mathrm{MeV}$.

The high-energy solar proton flux was enhanced for about a day. Obviously, the fluence, which is proportional to the area under the respective curves, was considerably greater for the 29
September 1989 event.

One of the conclusions in Shea and Smart (1990a) was that solar proton events occur in episodes of activity. This usually happens when one solar-active region produces a series of major flares with associated proton emission, as the active region rotates with the sun from the east to west limb. When regions such as this are near central meridian, the solar plasma emissions generally intersect the Earth a day or two after major flares, giving rise to major geomagnetic disturbances and often mid-latitude aurorae. At the same time, the traveling interplanetary shock wave associated with the plasma boundary re-accelerates the ambient solar proton flux in such a manner that, when the interplanetary shock arrives at the Earth, the solar particle flux increases as much as an order of magnitude for a few hours. In rare cases, particles up to $\mathrm{GeV}$ energies are involved (Levy, Duggal \& Pomerantz 1976). The fluences recorded during these episodes of activity can often be the major contribution to the solar proton fluence for a solar cycle.

Episodes of activity have occurred throughout solar cycle 22 with eight major sequences between March 1989 and June 1991. Table 1 presents a summary of the solar proton fluence for the past three solar cycles; fluences for the major events during the 22nd solar cycle are identified at the bottom of the table (Sauer, personal communication 1991, 1992). The solar proton fluence for the 22nd solar cycle has already exceeded the combined solar proton fluence for the 20th and 21st solar cycles, and is approaching the values for the 19th solar cycle. 
TABLE 1. Summary of Solar Proton Events for Solar Cycles 19, 20, and 21

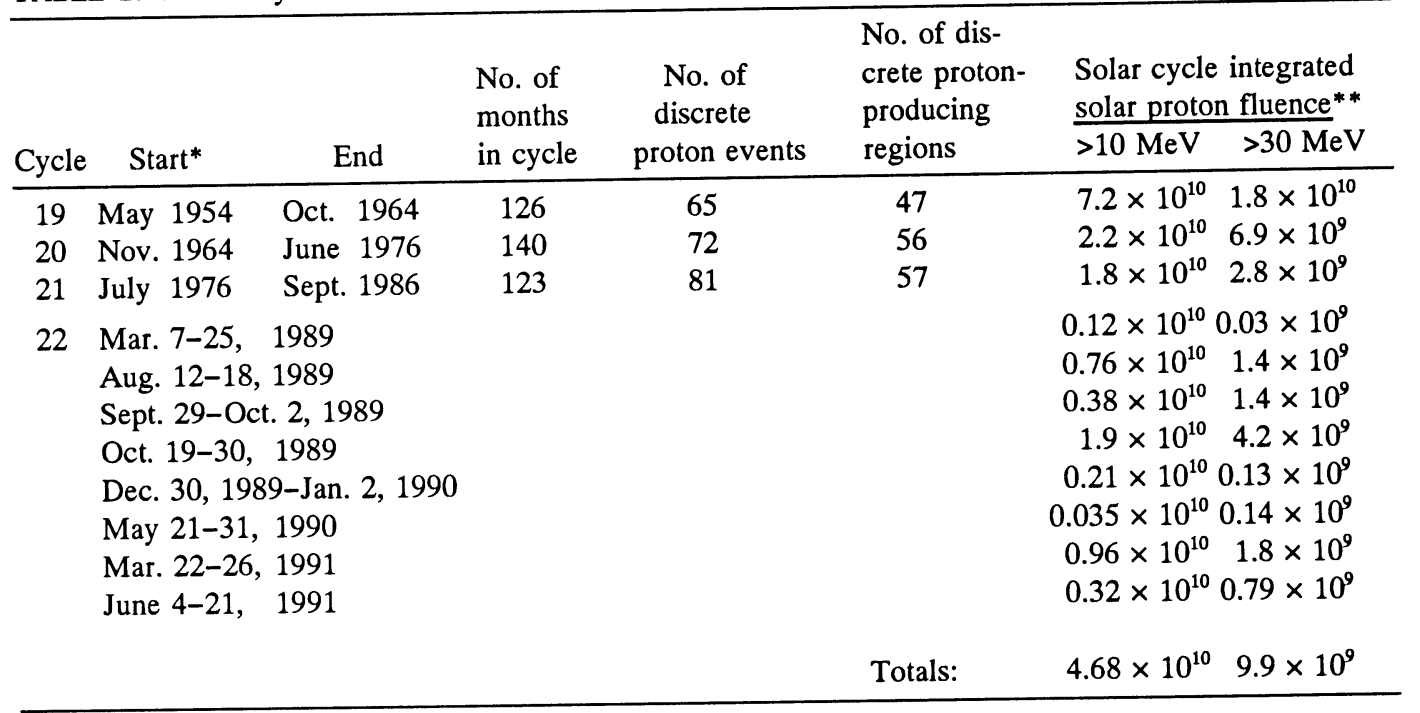

*The start of each solar cycle was selected as the month after the minimum in the smoothed sunspot number (McKinnon 1987).

**Fluence units are protons $\mathrm{cm}^{-2}$.

\section{EVENTS PRIOR TO SOLAR CYCLE 19}

In an attempt to identify solar proton events prior to the 19th solar cycle, Svestka (1966) interpreted vertical ionospheric sounding data during polar cap absorption (PCA) events, and compiled a list of PCA events probably associated with solar proton events between 1938 and 1955. In this work, he comments on the validity of each event, possible associated source solar flare and inferred solar flares. In an attempt to extend back further in time, we suggest a method of identifying periods of major proton fluences in the vicinity of the Earth, based upon the occurrence of major geomagnetic storms associated with episodes of solar activity across the solar disk.

We have previously argued (Smart \& Shea 1989; Shea \& Smart 1990a) that the shock-accelerated events (the July 1959 and the August 1972 episodes being outstanding examples) result in large particle populations observed at the Earth. Using the geomagnetic index of $\mathrm{Ap}^{*}>150$ as a proxy for identifying major solar proton events for the past three solar cycles, we successfully identified $90 \%$ of the major fluence events for the period, 1955-1986. In other words, direct correspondence exists between a major geomagnetic storm and a major solar proton fluence event. (The Ap* value, derived by Allen (1982), is the 24-hour running mean of the 3-hourly Ap value. Because the onset of geomagnetic disturbances is not always at $00 \mathrm{UT}$, and because most daily indexes are based on the 24-hour UT day, the Ap* values are an indication of the most severe 24-hour period of a geomagnetic disturbance.)

The good association between the presence of enhanced solar particle flux at the Earth and strong magnetic disturbances associated with solar flare episodes near the solar central meridian reflects the common source function, (i.e., solar activity). In fact, the presence of these particles, in association with episodes of solar activity as the source region crosses the solar disk, is reliable enough to be used as a predictor of geomagnetic disturbances. Although the technique described in the following paragraphs might identify many of the major fluence events, it cannot be used to postulate proton events that might have been associated with flares having source locations greater 
than $\sim 45^{\circ}$ from central meridian. Major proton events from west-limb solar activity similar to that of 23 February 1956, 4 May 1960 and 29 September 1989 would have to be inferred from other techniques.

From the geomagnetic records (Mayaud 1973), we can determine the dates of major geomagnetic disturbances for the past century. As a first approximation, one can assume that a major flare within $\pm 45^{\circ}$ of solar central meridian, followed within a day or two by a major geomagnetic disturbance, probably produced a solar particle event at the Earth. However, it is not possible to assemble a consistent record of these events over the past century, because the systematic flarepatrol records, organized by the International Astronomical Union, did not commence until January 1934.

In applying this "geomagnetic disturbance inference technique" to the "known" proton events prior to 1956, we have tested the procedure against the Svestka (1966) probable proton event list, which includes the four known ground-level events prior to 1956. For these associations, we used the probable flare location (Svestka 1966; Cliver et al. 1982), the aa* values and the sudden commencement lists of Mayaud (1973), and the Ap* values (Allen, personal communication 1989). From a combination of these records, we were able to associate all but three "strong" PCA events in Svestka's (1966) list with geomagnetic activity, and those three exceptions were events associated with possible source flare positions beyond $\pm 45^{\circ}$ of solar central meridian. Two of these "strong" PCA events were solar cosmic-ray ground-level events (7 March 1942 and 19 November 1949) emanating from source flares near the western limb of the sun, and were not associated with strong geomagnetic activity. Neither would the 23 February 1956 event be identified by this technique, because it was associated with a flare at $80^{\circ} \mathrm{W}$, and was not associated with a major geomagnetic storm.

\section{CONCLUSION}

Using the list of significant solar proton events from 1954 to 1986, we have found that the large fluence events at the Earth are usually associated with a sequence of solar activity near the central meridian of the sun and associated geomagnetic storms. This same phenomenon is true for the major events of the present solar cycle. From these results, we have suggested a method of identifying periods when major solar proton fluence events may have occurred throughout the past century. For this method, we use the geomagnetic field records and whatever solar observations are available. We emphasize that this method will not identify major proton events from flares on the western hemisphere of the Sun, because these flares typically do not usually generate a subsequent geomagnetic disturbance. We feel that this method can be further refined using other records of solar activity, such as the measurement of nuclides in polar ice cores.

\section{REFERENCES}

Allen, J. 1982 Some commonly used magnetic activity indices: Their derivation, meaning and use. In Proceedings of a Workshop on Satellite Drag, NOAA, ERL, Boulder, Colorado: 114-134.

Cliver, E. W., Kahler, S. W., Shea, M. A. and Smart, D. F. 1982 Injection onsets of $\sim 2 \mathrm{GeV}$ protons, $\sim 1 \mathrm{MeV}$ electrons, and $\sim 100 \mathrm{keV}$ electrons in solar cosmic ray flares. Astrophysical Journal 260: 362-370.

Feynman, J., Armstrong, T. P., Dao-Gibner, L. and Silverman, S. 1990 New interplanetary proton fluence model. Journal of Spacecraft and Rockets 27(4):

403-410.

Flückiger, E. O., Smart, D. F. and Shea, M. A. 1986 A procedure for estimating the changes in cosmic ray cutoff rigidities and asymptotic directions at low and middle latitudes during periods of enhanced geomagnetic activity. Journal of Geophysical Research 91(A7): 7925-7930.

Goswami, J. N., McGuire, R. E., Reedy, R. C., Lal, D. and Jha, R. 1988 Solar flare protons and alpha particles during the last three solar cycles. Journal of Geophysical Research 93(A7): 7195-7205. 
Levy, E. H., Duggal, S. P. and Pomerantz, M. A. 1976 Adiabatic Fermi acceleration of energetic particles between converging interplanetary shock waves. Journal of Geophysical Research 81(1): 51-59.

Lingenfelter, R. E. and Ramaty, R. 1970 Astrophysical and geophysical variations in $\mathrm{C} 14$ production. In Olsson, I. U., ed., Radiocarbon Variations and Absolute Chronology. Proceedings of the 12th Nobel Symposium. New York, John Wiley \& Sons, 513537.

Mayaud, P. N. 1973 A Hundred Year Series of Geomagnetic Data 1868-1967. IAGA Bulletin 33. Paris, IUGG Publication Office.

McKinnon, J. A. 1987 Sunspot Numbers 1610-1986 Based on the Sunspot Activity in the Years 16101960. UAG-95, NOAA, National Geophysical Data Center, Boulder, Colorado.
Shea, M. A. and Smart, D. F. 1990a A summary of major solar proton events. Solar Physics 127: 297320.

1990b Solar proton events - review and status. In Thompson, R. J., Cole, D. G., Wilkinson, P. J., Shea, M. A., Smart, D. F. and Heckman, G. R., eds., SolarTerrestrial Predictions. Proceedings of a Workshop at Leura, Australia. NOAA, Boulder, Colorado: 213-225.

Smart, D. F. and Shea, M. A. 1989 Solar proton events during the past three solar cycles. Journal of Spacecraft and Rockets 26(6): 403-415.

Svestka, Z. 1966 Proton flares before 1956. Bulletin of the Astronomical Institute of Czechoslovakia 17(5): 262-270. 